\title{
Ressecção de septo nasal devido ao espessamento congênito em potros
}

Nasal septum resection due congenital thickness in foals

\author{
Eduarda Maria Gomes das Neves Oliveira ${ }^{1 *}$; Peterson Triches Dornbusch²; \\ Ivan Deconto 3 ; Rubens Luiz Ferreira Gusso'; \\ Antônio Felipe Paulino de Figueiredo Wouk ${ }^{5}$; Renato Silva de Sousa ${ }^{6}$
}

\section{Resumo}

Obstruções nasais causadas por doenças do septo nasal são incomuns em cavalos e as principais causas são: malformações, lesões nas mucosas, neoplasias e trauma. $\mathrm{O}$ objetivo deste relato é descrever dois casos de obstrução nasal congênita em potros. Os sinais clínicos demonstravam redução progressiva do fluxo de ar bilateral, ruído respiratório e dispnéia intensa. A avaliação endoscópica não foi realizada pois em ambos os casos, não foi possível introduzir o equipamento na cavidade nasal devido à estenose bilateral. $\mathrm{O}$ exame radiográfico confirmou o aumento na espessura do septo nasal. Os animais foram submetidos à cirurgia de ressecção de septo. Para promover uma melhor condição respiratória a traqueostomia foi realizada nos dois potros antes da cirurgia. A técnica cirúrgica utilizada foi modificada da técnica descrita anteriormente DOYLE (2005). Inicialmente foi realizada a trepanação do osso nasal para osteotomia da porção caudal do septo, com auxilio de osteótomo modificado, sendo os limites dorsais e ventrais do septo removidos com uso de fio serra protegido por guias metálicos. Após a recuperação cirúrgica, nos dois casos, o exame clínico mostrou que o fluxo de ar estava normal e sem ruídos permitindo aos potros respirar normalmente. A traqueostomia cicatrizou por segunda intenção. Após oito meses os dois animais respiravam confortavelmente, estando aptos à atividade física. O exame histopatológico não demonstrou sinais de inflamação, neoplasia ou outro tipo de alteração histológica relacionada aos septos, sugerindo espessamento congênito.

Palavras-chave: Dispnéia, equino, ressecção de septo nasal

\footnotetext{
Abstract

Nasal obstructions caused by nasal septal diseases are uncommon in horses and the main causes are malformations, mucosal lesions, neoplasias and trauma. The aim of this case report is to describe two cases of nasal obstruction in foals. Clinical signs were progressive with reduction of air flow bilaterally, intense respiratory noise and dyspnea. Endoscopy evaluation was not performed because, in both cases, it was not possible to introduce the probe into the nasal cavity due to bilateral stenosis. Radiographic exam confirmed nasal septum thickness. Both animals were submitted to septum resection surgery. In order to promote a better respiratory condition, tracheotomy was performed in both foals prior the surgery. The surgical technique applied was modified from DOYLE technique described previously (2005). Briefly, nasal bone trephine role was initially done in order to do osteotomy of its caudal

1 Mestre pelo Programa Pós-Graduação Ciências Veterinárias, Universidade Federal do Paraná, UFPR. E-mail: eduardaoliveira@

2 Prof. Dr. Titular Departamento de Medicina Veterinária, UFPR. E-mail: petriches@gmail.br

Prof. Dr. Titular Departamento de Medicina Veterinária, UFPR. E-mail: deconto@ufpr.br

Msc. Médico Veterinário Autônomo. E-mail: rubensgusso@onda.com.br

Prof. Dr. Titular Departamento de Medicina Veterinária, UFPR. E-mail: fwouk@ufpr.br

Prof. Dr. Assistente Departamento de Medicina Veterinária, UFPR. E-mail: renatosousa@ufpr.br

Autor para correspondência
} ig.com.br 
portion. For osteotomy a modified osteotomy was used. The dorsal and ventral limits of the nasal bone were removed with a obstetric wire protected by metalical guide. After surgical recovery, in both cases, the clinical exam showed that the air flow was normal and without noise, allowing the foals to breathe normally. Tracheotomy healed by second intention. After eight months both foals breathed comfortably and they were suitable for physical activity. Histopathology evaluation did not show signs of inflammation, neoplasia, and any other histological changes, suggesting the thickness was congenital.

Key words: Dyspnea, equine, nasal septum resection

\section{Introdução}

As alterações de septo nasal causam resistência à passagem de ar na via aérea superior por invasão do meato nasal por lesões, malformação da cartilagem do septo, traumas e lesões proliferativas fúngicas ou neoplásicas (YARBROUGH et al., 1997; TROTTE et al., 2008). Este tipo de afecção é rara nos equinos, sendo que a principal causa é o trauma que pode promover o desvio ou espessamento do septo secundário à fratura (SHOEMAKER; WILSON; FRETZ, 2005). Em casos de espessamento congênito não foi descrito relação com predisposição de raça ou sexo (NICKELS, 1993; GAUGHAN; DEBOWES, 1993).

Os sinais clínicos relacionados à obstrução nasal são descritos como ruídos respiratórios, secreção nasal, diminuição do fluxo de ar em uma ou ambas as narinas e redução no desempenho atlético, podendo estar presentes ao nascimento ou em animais mais velhos (NICKELS, 1993). Os casos diagnosticados como anormalidades na cartilagem do septo nasal podem requerer a remoção cirúrgica principalmente quando o fluxo de ar está reduzido comprometendo a oxigenação do animal (SHARMA et al., 2010), sendo que em cavalos, a remoção do septo nasal é usualmente empregada para o alivio dos sinais clínicos, pois permite uma maior passagem de ar pelo meato nasal, diminuindo assim, a obstrução respiratória (YARBROUGH et al., 1997).

O diagnóstico é realizado por meio do histórico, sinais clínicos, exames de imagem endoscopia e radiografias, citológico e cultura da secreção nasal. O exame endoscópico é utilizado para determinar a integridade da cavidade nasal (ZOPPA et al., 2008).

Há cinco técnicas cirúrgicas relatadas para a ressecção do septo nasal: três delas utilizam a trepanação do osso nasal para a ressecção da porção caudal do septo, que em seguida utilizam dois fios serra que seccionam, cada um, a porção dorsal e ventral do septo em direção rostral sendo removida toda a sua extensão (DOYLE; FREEMAN, 2005). A quarta técnica realiza-se a incisão próxima à cartilagem alar visualizando e removendo apenas porções rostrais do septo, e a quinta técnica demonstra o acesso dorsal da cavidade, através da osteotomia do osso nasal para visualização do septo e sua remoção parcial (SHOEMAKER; WILSON; FRETZ, 2005).

O objetivo deste trabalho foi relatar dois casos de espessamento congênito de septo nasal diagnosticados e encaminhados para ressecção cirúrgica, avaliar a técnica cirúrgica adaptada empregada para o tratamento e as observações clínicas observadas.

\section{Material e Métodos}

Caso número 01: animal da raça Quarto-deMilha, com 2,5 anos de idade, destinado a provas de tambor e baliza, apresentava intensa dispnéia ao trote e ruído respiratório bastante evidente no início de doma. Os parâmetros clínicos ao exame estavam normais e a ausculta torácica sem alterações. Ao exame endoscópico não foi possível introduzir um endoscópio de $9 \mathrm{~mm}$ de espessura em nenhuma das narinas. Ao estudo radiográfico constatou-se espessamento acentuado bilateral do septo nasal.

Caso número 02: animal da raça Puro Sangue Inglês (PSI), com 7 meses de idade, que após o desmame passou a apresentar sinais de corrimento nasal bilateral, dispnéia e progressivo ruído 
respiratório observado ao pastejo. Ao exame clínico não foram verificados outros sinais e ausculta torácica estava normal. Ao exame endoscópico não foi possível introduzir o equipamento em nenhuma das narinas e o paciente foi encaminhado para exame radiográfico que demonstrou o espessamento bilateral do septo nasal.
As radiografias foram realizadas na posição dorso-ventral onde se observou o espessamento do septo nasal, sendo então, mensurados na porção mais espessa na imagem radiográfica obtendo-se os valores de 3,6 cm e 2,6 cm de largura nas porções mais largas, nos casos 1 e 2 respectivamente (Figura 1).

Figura 1. radiografia da região nasal em incidência dorso-ventral, se observa o septo nasal espessado, potro 7 meses (A); septo após a ressecção cirúrgica (B).

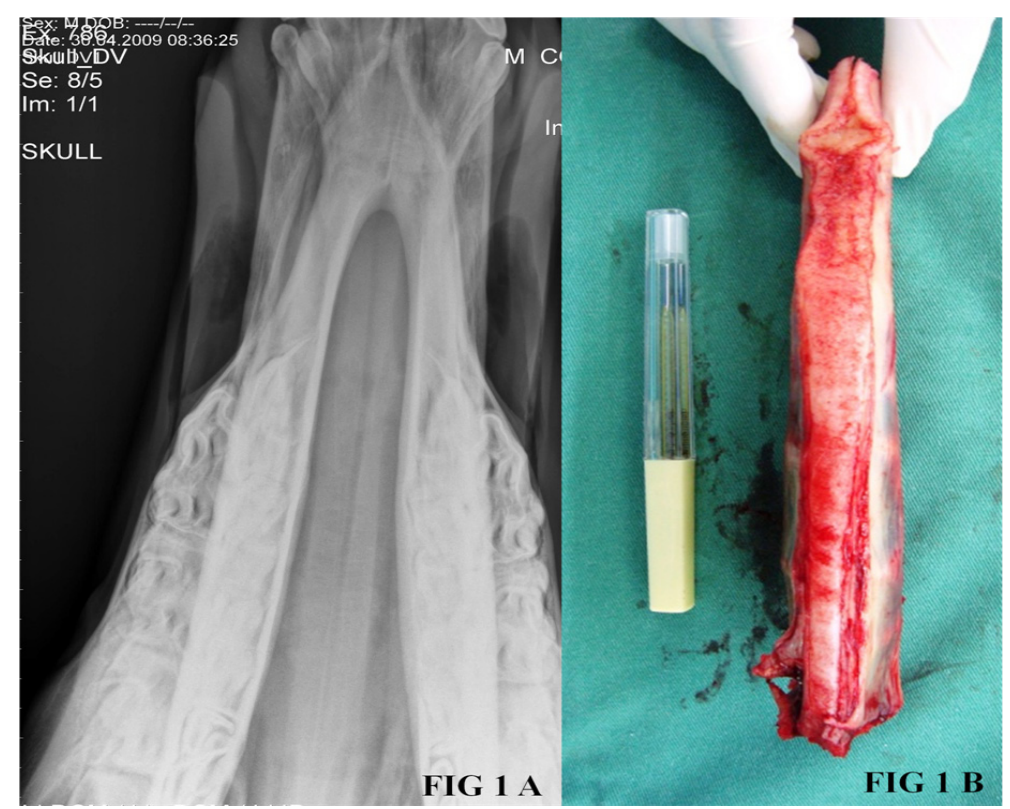

\section{Técnica cirúrgica}

Nos dois casos os animais foram encaminhados pelos veterinários responsáveis para realização de procedimento cirúrgico de ressecção do septo nasal.

Para diminuir o desconforto respiratório foi realizada traqueostomia temporária nos dois animais, sendo que o traqueotubo foi mantido até o quinto dia do pós-operatório.

Por se tratar de uma cirurgia extremamente cruenta com perda de grande volume sanguíneo, foram coletados cinco litros de sangue de animais doadores para ser utilizado no trans e pós-cirúrgico para a reposição volêmica.

Para a realização do procedimento cirúrgico os animais foram anestesiados, entubados via traqueostomia e posicionados em decúbito lateral esquerdo com as narinas levemente inclinadas abaixo da linha horizontal do decúbito, para facilitar a drenagem do sangue. Procedeu-se a depilação e antissepsia ampla da região dorsal para acesso ao osso nasal.

A técnica iniciou-se com a trepanação do osso nasal, na sua porção caudal, próximo a sínfise como osso frontal, o que permitiu o acesso à porção caudal do septo nasal. Após a retirada do fragmento circular de 14 milímetros de diâmetro, a porção caudal do septo foi seccionada na direção dorsoventral utilizando o osteótomo modificado, com sua extremidade cortante em forma de "V" 
direcionado caudalmente em um ângulo de 45 graus na linha média, (Figura 2). Na sequência foram inseridos, em cada narina, tubos metálicos com 40 centímetros por 5 milímetros de diâmetro, até o orifício da trepanação. Em cada um dos tubos foi introduzido uma das extremidades do fio serra. Direcionou-se o segmento do fio cortante através da porção caudal seccionada do septo, posicionando os tubos metálicos na porção ventral do septo nasal, iniciando a secção deste seguimento, até 5 $\mathrm{cm}$ da entrada das narinas. Durante o movimento de corte os tubos metálicos ficaram pressionados contra o assoalho da cavidade nasal, de ambos os lados, de forma a orientar a secção do septo o mais ventralmente possível. Em seguida, os tubos e o fio serra foram reposicionados no ponto de trepanação de forma semelhante à anterior para reiniciar a secção, desta vez, na porção dorsal do septo, tomando o cuidado em direcionar os tubos dorsalmente em contacto com o osso nasal. Finalmente, com auxílio de uma tesoura seccionou-se a porção rostral cartilaginosa do septo, permitindo a sua retirada pelo orifício nasal, tomando cuidado de manter ao menos 5 centímetros de septo rostralmente, para dar sustentação às narinas. A técnica cirúrgica foi adaptada de Doyle e Freeman (2005).

Figura 2. instrumental utilizado para retirada do septo nasal: fio serra introduzido dentro de tubos de aço com $5 \mathrm{~mm}$ de diâmetro. Ao centro observa-se o osteótomo modificado, reparar na extremidade cortante em forma de "V".

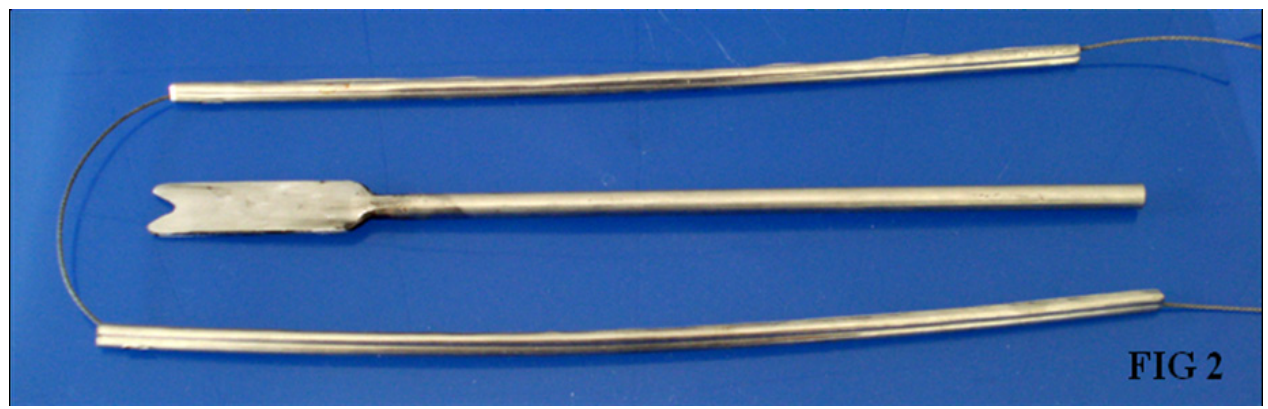

Assim que a abundante hemorragia foi observada iniciou a transfusão sanguínea sendo mantida até o controle e resolução da perda de sangue total, minimizando os efeitos hipovolêmicos.

A cavidade nasal foi preenchida com compressas cirúrgicas para realizar hemostasia compressiva. Foram aplicadas suturas para fechar as narinas evitando a saída das compressas. A medicação administrada no período pós-operatório foi penicilina benzatina por cinco dias associada antiinflamatório cetoprofeno por três dias.

Após as 48 horas de pós-cirúrgico as suturas nasais e as compressas foram removidas sem presença de sangramento, em ambos os casos. Quando ocluída a saída da traqueostomia, se verificou o fluxo de ar nas narinas, com os animais respirando confortavelmente e sem presença de ruídos. O traqueotubo foi removido no quinto dia do pós-operatório e a ferida cicatrizou por segunda intensão.

Após 03 meses o paciente número 01 retornou a doma sem ruídos respiratórios e em 12 meses encontrava-se competindo em provas de tambor e baliza.

O paciente número 02 retornou ao haras e aos dois anos encaminhado para treinamento em corridas de hipódromo. $\mathrm{O}$ exame endoscópico realizado, neste paciente, após 8 meses do procedimento cirúrgico mostrou completa cicatrização das estruturas seccionadas, sem tecido de granulação evidente e sem retorno dos sinais clínicos.

Nos dois casos, as amostras foram imersas em solução de formol $0,5 \%$ e processadas pelos métodos histológicos rotineiros até a inclusão em 
parafina e obtenção dos cortes transversais, os quais foram corados pelo método de hematoxilina-eosina (HE). Os estudos histopatológicos foram realizados em microscopia óptica não sendo visualizados sinais de inflamação, neoplasia ou outro tipo de alteração histológica relacionada aos septos, apenas o aumento da camada cartilaginosa do septo nasal sugerindo espessamento congênito.

\section{Discussão}

Os sinais observados nos dois casos condizem com os descritos na literatura sendo relatados por outros autores estrangeiros, entretanto não foram encontrados relatos de espessamento congênito em potros na literatura nacional.

Zoppa et al. (2008) descreveram que as lesões causadas por infecções fúngicas geralmente apresentam alterações inespecíficas, como extensão irregular e opacificação difusa o que diferiu das imagens radiográficas dos casos observados neste estudo, que mostraram o espessamento do septo nasal em toda extensão sem apresentar áreas irregulares. $\mathrm{O}$ diagnóstico radiográfico foi de grande importância para avaliar a extensão do espessamento do septo nasal e para a decisão da necessidade de intervenção cirúrgica.

Quanto ao posicionamento do animal na mesa cirúrgica o decúbito lateral é utilizado para facilitar a manipulação da região dorsal da cavidade nasal, porém, foi relatado por Shoemaker, Wilson e Fretz (2005) que a inclinação com a cabeça elevada a $45^{\circ}$, segundo autor facilita a visualização, entretanto nestes procedimentos foi utilizado o oposto, a cabeça levemente para inclinada para baixo facilitou a drenagem do sangue, desta forma evitando acúmulo nas vias aéreas caudais.

O procedimento cirúrgico por ser extremamente cruento necessita de habilidade do cirurgião para que o tempo trans-operatório seja o menor possível diminuindo o risco de morte do animal por hipovolemia. Por isso a coleta de sangue previamente foi importante para realização de transfusão durante o procedimento como descrito por Shoemaker, Wilson e Fretz (2005).

A traqueostomia temporária permitiu a sutura das narinas no pós-cirúrgico, possibilitando que o animal respire confortavelmente, conforme orientado por Doyle e Freeman (2005) e Yarbrough et al. (1997).

Nickels (1993) ao avaliar potros com idade inferior a seis meses que foram submetidos à ressecção do septo nasal, verificou em seu estudo, que um ano após o procedimento houve maior incidência de retorno dos sinais clínicos devido a distorções faciais, o que não observamos nestes dois casos, provavelmente, por serem animais maiores e mais velhos.

A técnica cirúrgica para ressecção do septo nasal dos "três arames" descrita por Doyle e Freeman (2005), um fio serra em cada porção do septo nasal, foi modificada utilizando-se tubos metálicos, e apenas um fio serra para a secção de cada uma das porções dorsal e ventral do septo nasal realizadas individualmente. Esta adaptação tornou a técnica mais rápida e fácil em guiar os fios metálicos por dentro da cavidade nasal, além de minimizar as lesões na mucosa e estruturas anatômicas adjacentes, evitando a formação exuberante de tecido cicatricial e granulação previamente descritas por Doyle e Freeman (2005) e, também permitiu uma secção mais regular e próxima à porção dorsal e ventral da cavidade nasal.

As complicações reportadas por Doyle e Freeman (2005) como epistaxe leve após remoção do tampão, celulites no local da traqueostomia, febre intermitente no pós-cirurgico, diarréia e obstrução das vias aéreas por coágulo de sangue não foram observadas.

Relatos de Tulleners e Raker (1983) e Sharma et al. (2010) descreveram casos de obliteração da cavidade nasal, e ao realizarem os exames histopatológicos não concluíram qual a etiologia, pois não visualizaram lesões histopatológicas 
como sinais de inflamação, infecção ou neoplasias, semelhante aos resultados deste estudo. Desta forma, o histórico e idade dos animais sugerem espessamento congênito da porção cartilaginosa do septo nasal, que se agravou com o desenvolvimento das estruturas da cabeça, como a causa mais provável destas afecções.

\section{Conclusão}

A técnica cirúrgica empregada de remoção de septo nasal espessado mostra-se viável em potros com mais de sete meses de idade, reduzindo a possibilidade dos efeitos colaterais como desvios laterais das narinas e/ou colabamento das vias respiratórias anteriores, permitindo o pleno restabelecimento da passagem de ar pela cavidade nasal e o retorno dos animais as suas funções esportivas.

\section{Referências}

DOYLE, A. J.; FREEMAN, D. E. Extensive nasal septum resection using a 3-wire method. Veterinary Surgery, v. 34, p. 167-173, 2005. Disponível em: <http:// onlinelibrary.wiley.com>. Acesso em: 22 fev. 2010.

GAUGHAN, E. M.; DEBOWES, R. M. Congenital diseases of the equine head. Veterinary Clinics of North America: Equine Practice, v. 9, p. 93-110, 1993. Disponível em: <http://www.ncbi.nlm.nih.gov/pubmed/ 8472207>. Acesso em: 20 fev. 2010.

NICKELS, F. A. Diseases of the nasal cavity. Veterinary Clinics of North America: Equine Practice, v. 9, p. 111121, 1993. Disponível em: < http://www.ncbi.nlm.nih. gov/pubmed/8472195>. Acesso em: 20 fev. 2010.
SHARMA, A.; THOMPSON, M. S.; SCHNABEL, L. V.; ASLI METE, A.; HACKETT, R. Imaging diagnosisEquine nasal septal thickening due to chronic chondritis. Veterinary Radiology \& Ultrasound, v. 51, n. 1, p. 65-68, 2010. Disponível em: <http://onlinelibrary.wiley.com>. Acesso em: 09 fev. 2010.

SHOEMAKER, R. W.; WILSON, D. G.; FRETZ, P. B. A dorsal approach for the removal of the nasal septum in the horse. Veterinary Surgery, v. 34, n. 6, p. 668-673, 2005. Disponível em: <http://onlinelibrary.wiley.com>. Acesso em: 22 fev. 2010.

TROTTE, M. N. de S.; SANTOS, I. B. dos; MIRANDA, L. H. M. de; AMORIM, A. R.; BORGES, J. R. J.; MENEZES, R. C. Histopatologia de lesões tumoriformes presentes na cavidade nasal de eqüídeos do Brasil. Ciência Rural, Santa Maria, v. 38, n. 9, p. 2535-2539, 2008. Disponível em: <http://www. scielo.br/scielo.php? script $=$ sci_arttext\&pid $=$ S0103$84782008000900019 \& \operatorname{lng}=$ en\&nrm $^{-}=$iso $>$. Acesso em: 09 de fev. 2010

TULLENERS, E. P.; RAKER, C. W. Nasal septum resection in the horse. Veterinary Surgery, v. 12, p. 41-47, 1983. Disponível em: <http://onlinelibrary.wiley.com>. Acesso em: 22 fev. 2010.

YARBROUGH, T. B.; CARR, E. A.; SNYDER, J. R.; HORNOF, W. J. Nasal septoplasty for correction of septal deviation in a foal. Veterinary Surgery, v. 26, n. 4, p. 340-345, 1997. Disponível em: <http://onlinelibrary. wiley.com>. Acesso em: 22 fev. 2010.

ZOPPA, A. L. V.; CRISPIM, R.; SINHORINI, I. L.; BENITES, N. R.; SILVA, L. C. L. C.; BACCARIN, R. Y. A.Obstrução nasal por granuloma fúngico em equino: Relato de caso. Arquivo Brasileiro de Medicina Veterinária e Zootecnia, Belo Horizonte, v. 60, n. 2, p. 315-321, 2008. Disponível em: <http://www. scielo.br/scielo.php? script $=$ sci_arttext\&pid $=\mathrm{S} 0102$ 09352008000200006\&lng=en\&nrm=iso>. Acesso em: 09 fev. 2010. 